\title{
Psychiatry in Bermuda
}

\author{
Karl Marlowe
}

Bermuda has one psychiatric hospital, which is organised along a programme model; an acute programme, a rehabilitation programme, a child and adolescent programme, a substance misuse programme and a learning disability programme. Bermuda has good resources and the majority of staff are trained in British hospitals. There has been a shift away from the stigma of mental hospitals towards a more community-oriented mental health service.

\section{History}

Bermuda comprises of an archipelago of tiny islands less than 22 square miles in total land mass, in the mid-Atlantic Ocean. It is therefore one of the most isolated places on earth. The warm Atlantic Gulf Stream maintains Bermuda's subtropical climate. The unique culture of Bermuda owes much to its geography and that it is the oldest British colony in the western hemisphere. Human settlement started in 1609 as a result of a British shipwreck, and this is said to have given Shakespeare the original story on which to base The Tempest.

At present Bermuda is a self-governing colony of the UK, but retains a governor appointed by the UK government. The population is 58480 with a population density of 3160 per square mile; the ethnic breakdown is of $61 \%$ Black and $39 \%$ nonBlack. The female life expectancy is 78 years, and 70 years for males (Population and Vital Statistics, 1996). The per capita income is within the top three in the world, with the average employment income being US\$32 171, with no income tax being levied (Facts \& Figures, 1996).

\section{Government/insurance funded services}

The Bermuda Hospital Board runs the two hospitals in Bermuda. King Edward VII Memorial Hospital, the general hospital, has 236 acute beds and 104 elderly long-stay beds. St Brendan's Hospital, the psychiatric hospital, has 25 acute beds and 96 long-stay and rehabilitation beds, this is in comparison to 29 acute beds and 148 long-stay and rehabilitation beds in 1991. The Montrose Substance Abuse Centre is part of the hospital out-patient treatment facilities. There are 100 medical doctors, 553 professional nurses and
22 dentists registered in Bermuda (Health, 1996). As well as the two hospital facilities, there are other Government agencies: The Physical Abuse Centre, Addiction Services, The National Drugs Commission. Child and Family Services and Teen Services (Health for Success, 1997).

\section{Private (non-Government funded) agencies}

Bermuda has a large private counselling sector. There are two employee assistance programmes (EAPs), as well as two other psychotherapy practices. There is a private Institute for Child and Family Health, and separate charities involved with AIDS and substance misuse. These private centres are well run by Bermudans and non-Bermudans, and there are two consultant psychiatrists working full-time in this sector.

\section{Health care}

St Brendan's Hospital, opened in 1835, is Bermuda's only in-patient treatment centre for the mentally ill and the mentally disabled. At present it is funded partly by private insurance (the initial 40 days of in-patient care) and by a government subsidy.

\section{In-patient care}

The hospital has an acute open ward (19 beds), a locked ward (six beds), an extended long-stay ward ( 26 beds), a psychiatric rehabilitation ward (19 beds) and four learning disability wards (total 51 beds). The acute bed occupancy has increased from $48 \%$ in 1991 to $79 \%$ in 1995 . during this time the number of admissions has increased from 204 to 263 (Health, 1996).

\section{Community care}

The general adult community mental health clinic is based at the general hospital. Referrals are taken from any member of the public, and it tends to be concerned relatives who phone the clinic to make a referral. A high percentage of self-referrals come with respect to reports for termination of pregnancy, as the Abortion Act requires a mental health assessment. Visits to the clinic have increased from 4920 to 5420 
between 1991 to 1995 (Health, 1996). Assessments are initially done by community psychiatric nurses (CPNs) or by junior medical staff.

There are three community psychiatric rehabilitation group homes, and a psychiatric day care facility run by the occupational therapy and the industrial therapy department. At present there is a weekly psychiatric clinic at the three prisons, and a weekly clinic at the Montrose Substance Abuse Centre.

The learning disability service is becoming more community orientated and there are four community group homes, with a provision to open a day hospital in the near future.

\section{Staff}

In 1971, the hospital board commissioned reports from Professor Jones, of Dalhouse University, and Professor Lishman, of London University; both recommended the establishment of four consultant psychiatrists to maintain an island-wide service. At present there are two general adult posts with coverage of the three prisons and substance misuse. In addition there is one post in adult rehabilitation and one post in child and adolescent psychiatry. There are three junior psychiatric posts with in-patient and outpatient responsibilities.

The other professional staff includes four clinical psychologists, three social workers, six mental welfare officers (CPNs with the legal authority to apply for detention under the Mental Health Act 1968), six CPNs, five occupational therapists, two industrial therapists, three recreation therapists, and two industrial therapist's aids. The hospital board employs all these staff.

\section{The Mental Health Act}

This 1968 Act has sections for emergency admission, admission for observation and treatment, admission for treatment, and similar forensic sections to the Mental Health Act (1983) for England and Wales. The main difference is a section for discharge from hospital conditional on accepting treatment, and if treatment is refused then detention for treatment is reinstated for an additional six months. Even though there is a Mental Health Act Review Tribunal and a high rate of formal admission, there is a very low rate of appeal to the tribunal.

\section{Mental illness}

There are very few cases of deliberate self-harm seen, but there are many referrals for adjustment disorder. The suicide rate was 8.5 per 100000 in 1991 and 3.4 per 100000 in 1996 (further details available from the author upon request). The prevalence for schizophrenia is similar to the
UK (approximately $1 \%$ of the adult population). but what is interesting is seeing more than four members of the same family with a psychotic illness and tracing the genetic links of this small genetic pool. It is difficult to quantify the prevalence of affective disorders because many patients are seen privately by the general practitioners, psychiatrists, counsellors or go abroad for treatment in Canada or the USA. The number of AIDS-related psychiatric disorders has increased as the number of deaths from AIDS has fallen (39 to 22, between 1994 and 1995), and this is in the context of an increase in incidence from 33 in 1990, with 48 new cases in 1995 (Health, 1996).

\section{Working in Bermuda}

It is necessary to obtain a permit before entering Bermuda to work. Wherever you work outside of the UK; you will have to pay tax if you return within 12 months (Going to Work Abroad, 1992). Consultants can negotiate a contract with the hospital management, while the junior medical staff are members of a collective union agreement which has been negotiated on their behalf. At present two of the three posts are eligible for up to one year of psychiatric training with the Royal College of Psychiatrists, as they hold Canadian certification (please check with the College before accepting a job on this proviso).

For any British-trained psychiatrists working in Bermuda, it is less taxing in more than one way, for as well as the similar Mental Health Act (modelled on the 1959 England and Wales Act), the prescribing practice is very similar.

After having worked in Bermuda for 16 months as a resident psychiatrist, I have found the experience of an allied but different service enlightening, and an understanding of different systems is vital as we take part in the process of change in the National Health Service.

\section{References}

GoING to WORK ABROAD? (1992) Personal Taxpayers Series IR58. London: Inland Revenue.

FACTS \& FIGURES (1996) Bermuda Goverment Statistics Department. Hamilton. Bermuda: Ministry of Finance.

Health (1996) Bermuda Digest of Statistics. Bermuda Statistics Department. Hamilton. Bermuda: Ministry of Finance.

HEALTH FOR SUCCESS (1997) A Comprehensive School Health Partnership Program. Hamilton. Bermuda Ministry of Health and the Ministry of Education.

Population \& VITAL Statistics (1996) Bermuda Digest of Statistics. Bermuda Statistics Department. Hamilton. Bermuda: Ministry of Finance.

Karl Marlowe, Registrar in Child Psychiatry. Child and Family Consultation Service, 1st Floor, Out-Patient Building. The Royal London Hospital (Whitechapel), London E1 1BB 